\title{
The Modal Moving Spotlight Theory
}

\author{
DANIEL DEASY \\ School of Philosophy, University College Dublin, Ireland \\ Forthcoming in Mind
}

\section{The moving spotlight}

Say that the Moving Spotlight Theory (MST) combines the following three theses:

A-THEORY: There is an absolute distinction between present and non-present times ${ }^{1}$

PERMANENTISM: Always, everything is always something

$\mathrm{A} \forall x \mathrm{~A} \exists y y=x$

SPOTLIGHT: Exactly one fundamental property is temporary, and it is monadic

A few remarks on these. First, not all self-described MSTists accept all of the above. For instance, Cameron (2015) defends a view that he describes as a version of MST which implies the falsehood of both Permanentism and Spotlight (on Cameron's view, there are temporary 'states of affairs', and there are many temporary fundamental properties - namely the fundamental ages of things). I do not mean to legislate here for the use of the name 'MST' - it is merely a label of convenience for the conjunction of the above theses.

Second, the A-theory is supposed to be read as a non-trivial thesis that would be rejected by those who hold that the predicate 'is present' is an indexical expression,

\footnotetext{
${ }^{1}$ I address the question of how MSTists should conceive of times in $\S 4$ below.
} 
analogous to 'is here'2 . However, note that it is supposed to be consistent with the A-theory that presentness understood as a property whose instances include times is a non-fundamental property.

Permanentism is reminiscent of the familiar thesis of 'Eternalism', but unlike that view - at least, as commonly understood - it does not entail a commitment to the concreteness of merely past and future things. For example, although Permanentism entails that if sometimes, something is a concrete dodo $(\mathrm{S} \exists x(C x \wedge D x))$ then something is sometimes a concrete dodo $(\exists x \mathrm{~S}(C x \wedge D x))$, it does not entail that there are concrete dodos $(\exists x(C x \wedge$ $D x)$ ); it is consistent with Permanentism that formerly concrete dodos are no longer concrete, or no longer dodos.

Spotlight is the view that there is exactly one temporary fundamental property, and that property is monadic. By 'property' in Spotlight (and throughout) I mean 'monadic or polyadic property', i.e. 'property or relation' (unless otherwise specified). A property is fundamental just in case it 'carves reality at the joints' or is 'perfectly natural' in the sense of Lewis (1983). I assume that part of the role of the fundamental properties is to provide a 'basis' for all properties, in the sense that every property can (in principle) be analysed in terms of some fundamental properties. And a property is temporary just in case sometimes, something sometimes has it and sometimes lacks it $(\mathrm{S} \exists x(\mathrm{~S} F x \wedge \mathrm{S} \neg F x))$.

Spotlight does not specify that the unique temporary fundamental property is presentness. That might seem odd, but there is a good reason for it: MST is compatible with several different views about the relation between the property or properties expressed by the word 'present' in English and the unique temporary fundamental property. For example, according to traditional versions of MST, the unique temporary fundamental property is a property of times, so that 'is present' as a predicate of times expresses the unique temporary

\footnotetext{
${ }^{2}$ Such as Sider (2001).
} 
fundamental property. However, MSTists could reject this traditional version of the view in favour of the view that e.g. the only bearers of the temporary fundamental property are spacetime points, and that times - conceived as sets or fusions of points - do not possess the unique temporary fundamental property. ${ }^{3}$ In that case, it is false that 'is present' as a predicate of times expresses the unique temporary fundamental property. Similarly, MSTists might think that 'is present' in English expresses a property that human beings possess when they are alive, but that no human being ever possesses the unique temporary fundamental property. In order to accommodate versions of MST on which 'present' in English typically expresses a non-fundamental temporary property (e.g. of times or of living people), it is better to characterise MST in terms of the weaker version of Spotlight above. Nevertheless, it is useful to have a name for the unique temporary fundamental property, whatever it may be. So, from now I will refer to it as 'fundamental presentness', being careful to distinguish it from any non-fundamental properties of presentness.

The view that fundamental presentness is the only temporary fundamental property is of course compatible with there being many temporary non-fundamental properties; and indeed, since MSTists should accept Temporalism (the view that there are temporary propositions - see $\S 2$ below), and if Temporalism is true then many ordinary predicates express temporary properties, MSTists should accept that many ordinary predicates express temporary non-fundamental properties whose analysis involves fundamental presentness. One way (but not the only way) to do to this is to accept the following semantic thesis:

ABOUT PRESENTNESS: For each property $F$ expressed by an ordinary predicate, there is a permanent relation $R$ such that $F$ is the temporary property of bearing $R$ to a

\footnotetext{
${ }^{3}$ See $\S 4$ below for the development of this view.
} 
present time ${ }^{4}$

About Presentness implies that ordinary predicates such as 'is sitting' express temporary properties of bearing certain permanent relations to a present time, so that e.g. the predicate 'is sitting' expresses the temporary (monadic) property of sitting-at a present time. Given About Presentness, the sentence (where ' $D x$ ' means ' $x$ is a dodo')

(1) It was the case that there is a dodo

$\mathrm{P} \exists x D x$

is equivalent to (where ' $D(x, y)$ ' means ' $x$ is a dodo-at $y$ '):

(2) It was the case that there is a dodo-at a present time

$\mathrm{P}(\exists x \exists t(\operatorname{Present}(t) \wedge D(x, t)))$

In what follows, I do not assume the truth of About Presentness given MST, but everything that follows is consistent with an acceptance of that view by MSTists.

Question: must those who accept MST as defined above also hold that some tense operators are fundamental, or can they provide a reductive analysis of the tense operators? (By 'tense operators' I mean sentence operators like 'It was the case that' ('P'), 'It will be the case that' (' $\mathrm{F}$ '), and 'It is the case at time $t$ that' ('at $t$ '). To say that a tense operator is fundamental is just to say that the property of propositions it expresses - such as being past or being future - is fundamental. ${ }^{5}$ ) In this paper, I argue that MSTists can provide a reductive

\footnotetext{
${ }^{4}$ This view is recommended to MSTists by Deasy (2015).

${ }^{5}$ I assume that sentence operators, and therefore tense operators in particular, express (higher-order) properties of propositions. On the higher-order approach to sentence operators, see e.g. Dorr (2016) and Fritz \& Goodman (2016, §3).
} 
analysis of the tense operators - in essence, a reductive account of change over time. I do this by developing a new analysis of the tense operators given MST which I call the Modal Analysis (so-called because it analyses tense operators in terms of a necessitation relation between propositions). The Modal Analysis can be thought of as a spelling-out in more fundamental terms of an analysis of the tense operators due to Parsons (2002), which I call the Counterfactual Analysis. I do not argue that the Modal Analysis represents the only, or even the best, way to combine the reduction of tense with MST. My aim is simply to present the Modal Analysis as one attractive way to deliver the reduction of tense given MST.

The rest of this paper is structured as follows: first (§2), I describe Parsons' (2002) Counterfactual Analysis. I argue that the Counterfactual Analysis meets an important condition for securing change over time which is accepted by many A-theorists. Then $(\S 3) \mathrm{I}$ introduce the Modal Analysis as a spelling-out, in more fundamental terms, of the Counterfactual Analysis. Next (§4), I combine this with a new account of times and fundamental presentness given MST. Finally (§5), I argue that the Modal Analysis shares the virtues of, and avoids the problems facing, the Counterfactual Analysis.

\section{The counterfactual analysis}

Parsons (2002, p. 1-2) describes 'the comprehensive A-theory which should be most plausible by the lights of the B-theorist: the "A-theory for B-theorists". Parsons' "A-theory for B-theorists' combines Permanentism and the A-theory with what I shall call the Counterfactual Analysis of the tense operators. It is useful to think of the Counterfactual Analysis as combining two separate analyses, as follows: first, what I shall call the Quantificational Analyses of the tense operators 'It was the case that' ('P') and 'It will be the case that' (' $\mathrm{F}$ ') (where ' $x<y$ ' means ' $x$ is earlier than $y$ '): 
For it to have been the case that $p$ is just for it to be the case that there is a time earlier than a present time at which $p$

$\mathrm{P} p \equiv \exists t_{1} \exists t_{2}\left(\operatorname{Present}\left(t_{1}\right) \wedge t_{2}<t_{1} \wedge \text { at } t_{2}(p)\right)^{6}$

For it to be such that it will be the case that $p$ is just for it to be the case that there is a time later than a present time at which $p$

$\mathrm{F} p \equiv \exists t_{1} \exists t_{2}\left(\operatorname{Present}\left(t_{1}\right) \wedge t_{1}<t_{2} \wedge\right.$ at $\left.t_{2}(p)\right)$

For example, given the Quantificational Analysis of 'P', sentence (1) above is equivalent to:

(3) There is a time earlier than a present time at which there is a dodo

$\exists t_{1} \exists t_{2}\left(\operatorname{Present}\left(t_{1}\right) \wedge t_{2}<t_{1} \wedge\right.$ at $\left.t_{2}(\exists x D x)\right)$

Second, Parsons' 'counterfactual' analysis of the tense operator 'It is the case at time $t$ that' ('at $t^{\prime}$ ):

AT-T (COUNTERFACTUAL): For it to be the case that at $t, p$ is for it to be the case that if $t$ were present, it would be that $p$

At $t(p) \equiv \operatorname{Present}(t) \square \longrightarrow p$

For example, if we apply Parsons' analysis of 'at $t$ ' to sentence (3) above, we get:

(4) There is a time earlier than a present time, and were that time present, there would be a dodo

\footnotetext{
${ }^{6}$ I follow Dorr (2016) in using ' $\equiv$ ' to indicate the giving of an analysis or 'real definition'.
} 
In short, according to the Counterfactual Analysis, what was is what would be were some past time present, and what will be is what would be were some future time present.

Parsons' Counterfactual Analysis seems to provide an attractive way to deliver the reduction of tense given MST. However, some might question whether the view that results from combining the Counterfactual Analysis with MST secures change over time. This is a difficult question, since different philosophers of time have different ideas - not always explicitly stated! - about what is required for there to be change over time. However, we can at least show that the combination of MST and the Counterfactual Analysis meets one widely-accepted (by A-theorists, at least) necessary condition for there being change over time.

Consider the thesis of Temporalism:

TEMPORALISM: For some $p$, sometimes $p$ and sometimes not- $p$ $\exists p(\mathrm{~S} p \wedge \mathrm{S} \neg p)$

We can think of Temporalism as the view that there are temporary propositions: propositions that are sometimes true and sometimes false, such as the proposition that I am sitting. ${ }^{7}$ An example of a non-temporary or permanent proposition is the proposition that I am sitting at $n$, where ' $n$ ' names this time: given that it is true, it is always true, and if it were false, it would always be false. ${ }^{8}$

\footnotetext{
${ }^{7}$ Not everyone will agree that this is an example of a temporary proposition, but that is because not everyone accepts that there are temporary propositions. See Brogaard (2012) and Bacon (2016) for recent defences of Temporalism.

${ }^{8}$ Following Prior (1971), Temporalism can be read as involving irreducibly higher-order quantification. In that case, Temporalism is compatible with the view that there are no such things (quantifying at the first-order) as propositions.
} 
Many A-theorists hold that there is change over time only if Temporalism is true.

For example, here is Prior:

To say that a change has occurred is to say at least this much: that something which was the case formerly is not the case now. That is, it is at least to say that for some sentence $p$ we have 'It was the case that $p$, and it is not the case that $p$ '. (Prior 1968, $\mathrm{p}$. 9) ${ }^{9}$

According to Prior, a change has occurred only if for some $p$, it was the case that $p$ and it is not the case that $p(\exists p(\mathrm{P} p \wedge \neg p))$. Generalising, we can say that change sometimes occurs i.e. that there is change over time - only if for some $p$, sometimes $p$ and sometimes not- $p$, i.e. only if Temporalism is true. Call this thesis Priorian Change:

PRIORIAN CHANGE: There is change over time only if Temporalism is true

MSTists who accept Priorian Change will want to ensure that their view secures the truth of Temporalism given any proposed reductive analysis of the tense operators. Given the Counterfactual Analysis, Temporalism reduces to:

(5) For some $p$, there is a time such that were it present, it would be that $p$, and there is a time such that were it present, it would be that not- $p$

$\exists p\left(\exists t_{1}\left(\operatorname{Present}\left(t_{1}\right) \square \longrightarrow p\right) \wedge \exists t_{2}\left(\operatorname{Present}\left(t_{2}\right) \square \rightarrow \neg p\right)\right)$

\footnotetext{
${ }^{9}$ Prior's use of the expression 'for some sentence $p$ ' here is apt to give the impression that his explanation of change involves first-order quantification over sentences, rather than higher-order quantification into sentence position. However, taking Prior's broader views into account - in particular, his seminal defence of primitive higher-order quantification in Prior (1971) - it seems plausible that this was just an unfortunate slip.
} 
And given MST, (5) is true. As a witness, consider the proposition that this time $n$ is present (although note that any time would do). There is a time $t_{1}$ - namely, $n-$ such that were $t_{1}$ present, that proposition would be true. And there is a time $t_{2}-$ e.g. some moment in $1996-$ such that were $t_{2}$ present, that proposition would be false (since it would still be true that only one time was present). It follows that given the combination of the Counterfactual Analysis with MST, Temporalism is true.

Some might object that change over time requires, not just the truth of Temporalism, but the fundamental truth of Temporalism. Given that Temporalism involves the tense operator 'It is sometimes the case that' ('S'), this objection implies that there is change over time only if some of the properties of propositions expressed by tense operators are fundamental. But why should we think that nothing changes if the properties of propositions expressed by tense operators are not fundamental? Unlike the view that nothing changes if the facts don't change (i.e. if Temporalism is false), the justification for this view is not at all clear.

We have seen that the Counterfactual Analysis provides MSTists with an analysis of the tense operators which secures the truth of Temporalism, and therefore meets the requirement for change expressed by Priorian Change. However, there are at least three good reasons for MSTists to look for an alternative analysis of the tense operators.

First, it is not clear that the Counterfactual Analysis secures the basic principles of standard linear tense logic. For example, consider Sometimes Elimination, the temporal analogue of the widely-accepted modal axiom ' 4 ' that what is possibly possible is possible $(\forall p(\diamond \diamond p \rightarrow \diamond p))$

SOMETIMES ELIMINATION: What is sometimes sometimes the case is sometimes the case 


$$
\forall p(\mathrm{SS} p \rightarrow \mathrm{S} p)
$$

Given the Counterfactual Analysis, Sometimes Elimination is equivalent to:

(6) $\forall p\left(\exists t_{1}\left(\operatorname{Present}\left(t_{1}\right) \square \rightarrow \exists t_{2}\left(\operatorname{Present}\left(t_{2}\right) \square \rightarrow p\right)\right) \rightarrow \exists t_{3}\left(\operatorname{Present}\left(t_{3}\right) \square \rightarrow p\right)\right)$

But the truth of (6) does not follow from any standard principles of counterfactual logic. It would be nice to find an analysis of the tense operators that secures standard tense-logical axioms such as Sometimes Elimination given MST.

Second, given that counterfactuals are not fundamental, the Counterfactual Analysis does not provide an analysis of sentences of the form 'at $t(p)$ ' in fundamental terms given an analysis of $p$ in fundamental terms. It would be nice to find an analysis of the tense operators that does.

Third, the Counterfactual Analysis faces a problem of circularity. In particular, it appears to be necessary to use temporal notions in order to provide a reading of the relevant counterfactuals on which the Counterfactual Analysis is extensionally adequate. To see this, consider that given the combination of the Counterfactual Analysis with MST, the truth of

(7) Jones was dancing

requires the truth of

(8) There is a past time $t$ such that were $t$ present, Jones would be dancing 
On the standard account of the semantics of counterfactuals, sentences of the form 'Were it the case that $p$, it would be the case that $q^{\prime}$ are true just in case $q$ is true in all of the $p$-worlds 'closest to' - i.e. most relevantly similar to - the actual world. ${ }^{10}$ In that case, given the combination of Counterfactual Analysis with MST, the truth of (7) requires the truth of

(9) There is a past time $t$ such that in all of the worlds closest to the actual world in which $t$ is present, Jones is dancing

But note that even if it is actually the case that Jones is dancing at a past time, it could have failed to be the case that Jones is dancing at a past time. For example, holding fixed the actual permanent relations between times, Jones could have failed to be dancing at any past time; or, the actual permanent relations between times could have been different such that all of the times at which Jones is actually dancing are future times. ${ }^{11}$ In 'possible worlds' terms: even if in the actual world Jones is dancing at a past time $t$, there are (merely) possible worlds in which Jones is not dancing at $t$ (or at any other past time). In that case, in order to secure the truth of (7) given MST, defenders of the Counterfactual Analysis must somehow exclude worlds in which Jones is not dancing at any of the times that are actually past from the set of relevantly-similar-to-actual worlds mentioned in the antecedent of (9). More generally, the Counterfactual Analysis only works given MST on condition that the actual permanent facts - e.g. truths about the actual permanent relations between individuals and times, such as that Jones bears the permanent dancing-at relation to a certain time $t$-are held fixed in the assessment of the relevant counterfactuals. However, in that case the Counterfactual Analysis is ultimately circular, as what it is for a fact to be permanent is for it to be always true, and

\footnotetext{
10 See e.g. Stalnaker (1968) and Lewis (1973).

${ }^{11}$ See Dorr \& Goodman (2020) for some relevant work on the interaction between tense and modality.
} 
being always true is one of the properties of propositions for which the Counterfactual Analysis is supposed to provide an analysis. ${ }^{12}$ Let us call this the 'circularity problem' for the Counterfactual Analysis.

\section{The modal analysis: introduction}

The best way to introduce the Modal Analysis is to consider the question of whether defenders of the Counterfactual Analysis can find some way of characterising the permanent facts given MST without mentioning their permanence. And it seems they can: as we saw in $\S 1$ above, according to MST, fundamental presentness is the unique temporary fundamental property. It follows that given MST, if a fact is not about fundamental presentness - in the sense of being a conjunction of attributions to things (of the appropriate type) of fundamental first- or higher-order properties other than fundamental presentness and their negations - then it is permanent. In that case, a defender of the Counterfactual Analysis could characterize the relevant reading of the counterfactuals given MST as that on which the facts not about fundamental presentness are held fixed - so that, for example, (7) ('Jones was dancing') is true just in case there is a past time $t$ such that holding fixed the facts not about fundamental presentness, were $t$ present, Jones would be dancing.

However, this idea naturally suggests a more fundamental analysis of 'at $t$ ' given MST, as follows (where ' $C p$ ' means ' $p$ is not about fundamental presentness' in the sense described above, and 'Present $(x)$ ' means ' $x$ is the present time'):

AT-T (NECESSITATION): For it to be the case that at $t, p$ is for it to be the case that $p$ is necessitated by some true proposition $q$ not about fundamental presentness and the proposition that $t$ is the present time

\footnotetext{
${ }^{12}$ On the standard account, $\mathrm{A} p$ reduces to $\neg \mathrm{S} \neg p$, and $\mathrm{S} p$ reduces to $\mathrm{P} p \vee p \vee \mathrm{F} p$.
} 
At $t(p) \equiv \exists q(q \wedge C q \wedge \square((q \wedge \operatorname{Present}(t)) \rightarrow p))^{13}$

Or, equivalently:

AT-T (COMPATIBILITY): For it to be the case that at $t, p$ is for it to be the case that $p$ is compatible with any true proposition $q$ not about fundamental presentness and the proposition that $t$ is the present time

At $t(p) \equiv \forall q((q \wedge C q) \rightarrow \diamond(p \wedge q \wedge \operatorname{Present}(t)))$

Call the combination of the Quantificational Analyses of 'P' and ' $F$ ' $(\S 2)$ with either of the above analyses of 'at $t$ ' the Modal Analysis. ${ }^{14}$ Informally, the Modal Analysis says that something is true at a time $t$ just in case it is necessitated by some permanent fact and the proposition that $t$ is the present time; or equivalently, just in case it is compatible with the conjunction of any permanent fact and the proposition that $t$ is the present time.

To see that the Necessitation and Compatibility analyses of 'at $t$ ' above are equivalent, notice that if $p$ is compatible with any true proposition $q$ not about fundamental presentness and the proposition that $t$ is the present time (i.e. if it is true that $\forall q((q \wedge C(q)) \rightarrow$ $\diamond(p \wedge q \wedge \operatorname{Present}(t))))$, then in particular, $p$ is compatible with the fact $q$ not about

\footnotetext{
${ }^{13}$ Whether the ' $\square$ ' here should be interpreted as expressing 'metaphysical' necessity, 'broad' necessity (see Bacon 2018) or some other kind of necessity is a question on which I remain neutral (along with the question of whether metaphysical necessity is broad necessity). However, see note 24 below for a reason to think that it expresses something narrower than either metaphysical or broad necessity.

${ }^{14}$ It is important that the 'facts not about fundamental presentness' in these analyses are characterised as above in terms of attributions of fundamental first- or higher-order properties other than fundamental presentness. For example, suppose that the believing relation between individuals and propositions is a fundamental higher-order relation not supervenient on any other fundamental relations (as it might be according to some forms of property dualism), and that 'Jones believes that fundamental presentness is interesting' is true. Then given the tenselogical axiom Sometimes Introduction $(\forall p(p \rightarrow \mathrm{S} p))$, 'Sometimes, Jones believes that fundamental presentness is interesting' is true. Given the Modal Analysis, this is equivalent to: 'For some time $t$, the proposition that Jones believes that fundamental presentness is interesting is necessitated by some true proposition $q$ not about fundamental presentness and the proposition that t is the present time'. But this sentence is true - and therefore a failure of Sometimes Introduction is avoided - only if the facts 'not about fundamental presentness' include attributions of fundamental higher-order relations such as the believing relation.
} 
fundamental presentness that fully specifies what there is and what fundamental first- or higher-order properties other than fundamental presentness are instantiated by things of the appropriate type; and any such truth, when combined with a proposition that fully specifies which things have the remaining fundamental property (of fundamental presentness), is maximally strong, and hence necessitates everything with which it is compatible (and therefore necessitates $p$ ).

Readers will notice that the above argument relies on the significant premise that everything supervenes on the distribution of fundamental properties, or in other words, that 'truth supervenes on being' (TSB). That should come as no surprise: given that the Modal Analysis requires there to be a well-defined operation of what would be the case were some other time present, MSTists who accept the Modal Analysis must accept some version of TSB. ${ }^{15}$ TSB is often understood as the view that necessarily, all truths are necessitated by truths about what there is (quantifying at the first-order) and what fundamental first-order properties are instantiated by objects. ${ }^{16}$ However, this 'objectual' version of TSB is incompatible with the view that there are fundamental higher-order relations that do not supervene on the pattern of instantiation of fundamental first-order properties by objects. ${ }^{17}$ MSTists who wish to maintain consistency with such views can instead accept the following 'higher-order' version of TSB:

\footnotetext{
${ }^{15}$ As a referee for this journal pointed out to me, there being a well-defined operation of what would be the case were $p$ the case (for any $p$ ) could be secured by an acceptance of Conditional Excluded Middle ( $\forall p \forall q(p \square \rightarrow q \vee$ $p \square \rightarrow \neg q)$ ) without assuming TSB. However, in that case the Modal Analysis would rely on counterfactuals, and avoiding counterfactuals is (as we have seen) part of the raison d'être of the Modal Analysis.

${ }^{16}$ See e.g. Bigelow (1998) and Lewis (1994, p. 473).

${ }^{17}$ An example of such a view is that defended by Hossack (2007). According to Hossack, the higher-order knowledge of relation between individuals and true propositions is fundamental.
} 
TSB (HO): Necessarily, all truths are necessitated by truths about what there is (of any type) and what fundamental first- or higher-order properties are instantiated by things of the appropriate type ${ }^{18}$

A question that might occur to some readers is whether the Modal Analysis is really distinct from, or is merely a version of, the Counterfactual Analysis. In particular, one might think that counterfactuals are contextually flexible enough that there is an interpretation of the Counterfactual Analysis on which it is equivalent to the Modal Analysis. Whether or not that is the case - it is at least not obviously the case - is not a question I will address here; but for convenience, I will continue to speak as if the analyses are distinct.

\section{The modal analysis: times}

Like the Counterfactual Analysis, the Modal Analysis relies on the notion of a time's being present given MST. But how should MSTists conceive of times, and what is it for a time to be present given MST? As an anonymous referee for this journal pointed out to me, how MSTists answer these questions matters as to whether the Modal Analysis delivers the basic principles of standard linear tense logic.

For example, suppose that according to MST, necessarily, times are hypersurfaces, and necessarily, a time is present just in case it possesses fundamental presentness. Now suppose that MSTists also accept the 'principle of recombination' according to which any distribution of fundamental properties is (metaphysically) possible, and therefore any distribution of fundamental presentness is possible. ${ }^{19}$ In that case, given MST, the (false) proposition that I possess fundamental presentness is possibly true. Moreover, that

\footnotetext{
18 See especially Dorr $(2019, \S 4.4 .1)$.

${ }^{19}$ On the principle of recombination, see e.g. Lewis (1986, pp. 87-88).
} 
proposition is compatible with the proposition $q$ not about fundamental presentness that fully specifies the distribution of fundamental non-presentness properties and the proposition that $t$ is the present time (i.e. that $t$ possesses fundamental presentness), for any $t$. It follows by the Necessitation analysis of 'at $t$ ' that there is no time $t$ such that at $t$, I do not possess fundamental presentness. But then, given the Quantificational Analysis of 'S', we have a failure of the tense-logical axiom Sometimes Introduction $(\forall p(p \rightarrow \mathrm{S} p))$ : I do not possess fundamental presentness, but it is not the case that sometimes, I do not possess fundamental presentness. $^{20}$

A second problem arises given the possibility that there is no unique present time, either because more than one hypersurface possesses fundamental presentness or no hypersurface possesses fundamental presentness (e.g. because there are no hypersurfaces). In that case, given MST, it is not necessary that there is a unique present time. However, by the Necessitation analysis of 'at $t$ ', it is necessarily the case that for any time $t$, at $t$, there is a unique present time; from which it follows given the Quantificational Analysis of 'A' that necessarily, always, there is a unique present time. Hence given MST, it is necessarily always the case that there is a unique present time, but it is not necessarily the case that there is a unique present time; which given standard modal reasoning entails that it is not necessarily the case that if there is always a unique present time then there is a unique present time. We have, therefore, a failure of the necessity of an instance of the tense-logical axiom Always Elimination $(\forall p(\mathrm{~A} p \rightarrow p))$.

The two problems for MSTists described above arise from the combination of the principle of recombination with the traditional view of times and fundamental presentness given MST. MSTists can avoid these problems by rejecting the traditional view of times and

\footnotetext{
${ }^{20}$ An analogous argument can be used to show that the possibility of my possessing fundamental presentness generates a failure of Always Elimination $(\forall p(\mathrm{~A} p \rightarrow p))$ given the Compatibility analysis of 'at $t$ '.
} 
fundamental presentness given MST. An attractive alternative picture is that hypersurfaces are sets of spacetime points, and fundamental presentness is a property, not of hypersurfaces, but of spacetime points. Call the latter view Pointy Presentness:

POINTY PRESENTNESS: Fundamental presentness is a (temporary) property of spacetime points

Given Pointy Presentness, it is natural for MSTists to think of the present time as the set of all and only the spacetime points that possess fundamental presentness, which we assume form a hypersurface; and past and future times as hypersurfaces (i.e. sets of points) parallel to the hypersurface formed by the points that possess fundamental presentness. However, a lesson of the problems for the traditional view described above is that given the principle of recombination, MSTists need to be flexible about what the present time could be. For that reason, given Pointy Presentness, MSTists should define 'present time' is as follows (where 'Present ${ }_{F}(x)$ ' means ' $x$ possesses fundamental presentness'):

PRESENT TIME (MST): For a time $t$ to be present is just for $t$ to be the set of all and only the things that possess fundamental presentness $\forall t\left(\operatorname{Present}(t) \equiv t=\left\{x: \operatorname{Present}_{F}(x)\right\}\right)$

On this view, the present time is the unique set of all and only the things that possess fundamental presentness, which given Pointy Presentness are spacetime points and, we assume, form a hypersurface, ${ }^{21}$ and past and future times are hypersurfaces (i.e. sets of

\footnotetext{
${ }^{21}$ The present time does not itself possess fundamental presentness on this view, just as e.g. a set of electrons is not itself an electron. It follows that on this view, presentness as a property of times is a non-fundamental temporary property.
} 
points) parallel to the hypersurface formed by the things that possess fundamental presentness. Given this definition of 'present time', 'time' can then be defined as follows:

TIMES (MST): For some $x$ to be a time is just for $x$ to be the set of all and only the things that possess fundamental presentness, or to be a set of spacetime points forming a hypersurface $h$ which is parallel to some other hypersurface $h^{*}$, such that $h^{*}$ is the set of all and only the things that possess fundamental presentness

It is worth drawing attention to some of the consequences for MSTists of the above view of times and of fundamental presentness.

First, it avoids the problems described above arising from the combination of the traditional view of times and fundamental presentness given MST with the principle of recombination. As for the first problem: given that I do not actually possess fundamental presentness and that set-membership facts are necessary, the proposition that I possess fundamental presentness is incompatible with the proposition $q$ not about fundamental presentness that fully specifies the distribution of fundamental non-presentness properties and the proposition that $t$ is the present time (i.e. that $t$ is the set of all and only the things that possess fundamental presentness), for any $t$. It follows that I do not possess fundamental presentness, and for any time $t$, at $t$, I do not possess fundamental presentness. Hence the failure of Sometimes Introduction is avoided.

As for the second problem: given that necessarily, there is a set of all and only the things that possess fundamental presentness (no matter how many or how few fundamentally present things there may be), it follows that necessarily, there is a unique present time. Hence the failure of the necessity of the relevant instance of Always Elimination is also avoided.

Second, the view lets MSTists adopt a picture of what the fundamental properties 
other than fundamental presentness are that is in line with the standard B-theory, i.e. so that those other fundamental properties don't privilege any special direction in spacetime.

Third, given that parallel to is necessarily an equivalence relation on the set of times, it follows from the view that facts about which hypersurfaces are times are permanent. It also follows that necessarily, every time is a time at every time. Call this thesis Eternal Temporality:

ETERNAL TEMPORALITY: Necessarily, everything that is a time is a time at every time $\square \forall x(\operatorname{Time}(x) \rightarrow \forall t$ at $t(\operatorname{Time}(x)))$

Fourth, it follows from the view that in order for there to be multiple times, fundamental presentness has to be 'well-behaved', in the sense that its instances form an appropriate hypersurface; possibilities in which fundamental presentness is not well-behaved in that sense count as possibilities in which there is only one time.

Some readers may wonder how the view of times and fundamental presentness described above carries over to a general relativistic (GR) setting. This is not a question I wish to address in detail here; however, it is nevertheless worth making a few points about the issue. First, the view at least provides a useful way to frame the question, as follows: is there a relation between regions of spacetime given GR that can play the role that parallelism plays in the definition of 'time' above? It may be that in certain 'well-behaved' GR spacetimes, there is; in which case, the view may be carried over to such settings. (Note that the parallel to relation mentioned in the definition of 'time' above does not have to be a fundamental relation; however, it does have to be an equivalence relation.) Second, notice that no version of MST (as it has been defined here) can be fully consistent with either the special or general relativistic views of fundamental temporal reality, in the sense of fully 
agreeing with those views (or at least, with their philosophical interpretations) as to the nature of fundamental temporal reality. The reason is that MSTists are committed to Spotlight, and therefore to there being a fundamental monadic property in addition to the fundamental properties posited to spacetime physics. Of course, some philosophers will reject MST on this basis. But it also has implications for the question of whether the view of times and fundamental presentness described above can be carried over to a GR setting: given that MSTists already posit a fundamental property in addition to those posited by spacetime physics, they may be content to marry their view with GR by simply positing an additional fundamental permanent relation that can play the role in a GR setting that parallelism plays in the definition of 'time' above. ${ }^{22}$

\section{The modal analysis: virtues}

The Modal Analysis shares the virtues of, as well as avoiding the problems facing, the Counterfactual Analysis.

Like the Counterfactual Analysis, the Modal Analysis secures the truth of Temporalism given MST, and therefore meets the requirement for change expressed by Priorian Change. Given the Modal Analysis, Temporalism $(\exists p(\mathrm{~S} p \wedge \mathrm{S} \neg p))$ reduces to:

\footnotetext{
${ }^{22}$ See Bacon (2016) for the development of a view that aims to combine Temporalism with the special and general relativistic views of fundamental temporal reality. It is worth briefly describing some of the ways in which Bacon's theory both resembles and differs from the version of MST defended in this paper. First, both theories are Temporalist and A-theoretic (in the sense of positing an absolute distinction between present and non-present times). Both theories also treat being a time and being present (as a property of times) as nonfundamental properties. However, whereas according to Bacon's view, all of the fundamental facts are permanent, according to the theory defended in this paper, some fundamental facts - in particular, facts about fundamental presentness - are temporary. Given that Bacon also holds that all facts supervene on the fundamental facts and that some (non-fundamental) facts are temporary, it follows that on Bacon's view, there are temporary (metaphysical) necessities (see Bacon 2016, note 27); whereas the theory defended in this paper is consistent with, and in fact implies, the view that necessity implies permanence (see especially Dorr \& Goodman 2020).
} 
(10) For some $p$ : there is a time $t_{1}$ such that the proposition that $t_{1}$ is the present time together with some fact $q$ not about fundamental presentness necessitates $p$; and there is a time $t_{2}$ such that the proposition that $t_{2}$ is the present time together with some fact $r$ not about fundamental presentness necessitates not- $p$

$\exists p\left(\exists t_{1}\left(\exists q\left(q \wedge C q \wedge \square\left(\left(q \wedge \operatorname{Present}\left(t_{1}\right)\right) \rightarrow p\right)\right)\right) \wedge \exists t_{2}\left(\exists r\left(r \wedge C r \wedge \square\left(\left(r \wedge \operatorname{Present}\left(t_{2}\right)\right)\right.\right.\right.\right.$ $\rightarrow \neg p))))$

And (10) is true given MST. For example, let $p$ be the proposition that $n$ is present, where ' $n$ ' names this time (although note that any time would do). There is a time $t_{1}-$ namely, $n-$ such that the conjunction of some true proposition not about fundamental presentness (e.g. the proposition that $n=n$ ) with the proposition that $t_{1}$ is present necessitates $p$, and there is a time $t_{2}$ - for example, some moment in 1996 - such that the conjunction of some true proposition not about fundamental presentness (e.g. the proposition that $n=n$ ) with the proposition that $t_{2}$ is present necessitates not- $p$.

The Modal Analysis also avoids the problems for the Counterfactual Analysis described in $\S 2$ above that motivated the search for an alternative to that view. First, it provides a fundamental analysis of 'at $t(p)$ ' given an analysis of $p$ in fundamental terms. This is especially clear if we take ' $\square$ ' in the Modal Analysis as fundamental, or if we treat the entailment relation between propositions as fundamental.

Second, it secures the basic principles of standard linear tense logic. For reasons of space, I focus on just two here: Always Elimination $(\forall p(\mathrm{~A} p \rightarrow p))$, the temporal analogue of the modal axiom ' $T$ ' that what must be the case is the case $(\forall p(\square p \rightarrow p))$; and Sometimes 
Elimination $(\forall p(\mathrm{SS} p \rightarrow \mathrm{S} p))$, which was introduced in $\S 2$ above in relation to the

Counterfactual Analysis. ${ }^{23}$

We begin with Always Elimination:

ALWAYS ELIMINATION: What is always the case is the case

$\forall p(\mathrm{~A} p \rightarrow p)$

The Modal Analysis secures the necessary truth of Always Elimination given MST. To see this, we begin by noting that given the Modal Analysis, Always Elimination is equivalent to:

(11) For any $p$ : if for any time $t, p$ is necessitated by some true proposition $q$ not about fundamental presentness and the proposition that $t$ is the present time, then $p$ $\forall p(\forall t(\exists q(q \wedge C q \wedge \square((q \wedge \operatorname{Present}(t)) \rightarrow p))) \rightarrow p)$

It is easy to see that (11) is necessarily true given MST. Take any proposition $p$ : necessarily, if for any time $t, p$ is necessitated by some true proposition $q$ not about fundamental presentness and the proposition that $t$ is the present time, then in particular, for this time $n, p$

\footnotetext{
${ }^{23}$ In order to secure an S5 logic for 'Always', we would need the following principles in addition to Always Elimination and Sometimes Elimination:
}

ETERNALISATION: If $p$ is a theorem then A $p$ is also a theorem

ALWAYS DISTRIBUTION: $\forall p \forall q(\mathrm{~A}(p \rightarrow q) \rightarrow(\mathrm{A} p \rightarrow \mathrm{A} q))$

TEMPORAL B: $\forall p(p \rightarrow \mathrm{AS} p)$

Eternalisation is the temporal analogue of the modal axiom 'Necessitation', according to which if $p$ is a theorem then $\square p$ is also a theorem. Eternalisation follows from Necessitation given the principle that what must be the case is always the case $(\forall p(\square p \rightarrow \mathrm{A} p))$, which follows immediately from the Modal Analysis. Always Distribution is the temporal analogue of the modal axiom ' $\mathrm{K}$ ' that if necessarily, $p$ only if $q$, then necessarily $p$ only if necessarily $q(\forall p \forall q(\square(p \rightarrow q) \rightarrow(\square p \rightarrow \square q)))$. Finally, Temporal $B$ is the temporal analogue of the modal axiom ' $\mathrm{B}$ ' that what is the case must be possible $(\forall p(p \rightarrow \square \diamond p))$. I leave it as an exercise for readers to show that the Modal Analysis secures the necessary truth of Always Distribution and Temporal B given MST. 
is necessitated by some true proposition $q$ not about fundamental presentness and the proposition that $n$ is the present time. Given that according to MST, the proposition that $n$ is the present time (i.e. that all and only the members of $n$ possess fundamental presentness) is true, it follows that $p$.

Next, Sometimes Elimination:

SOMETIMES ELIMINATION: What is sometimes sometimes the case is sometimes the case $\forall p(\mathrm{SS} p \rightarrow \mathrm{S} p)$

Given the Compatibility analysis of 'at $t$ ', 'SSp' reduces to:

(12) $\forall q\left((q \wedge C q) \rightarrow \exists t_{1} \diamond\left(\operatorname{Present}\left(t_{1}\right) \wedge q \wedge \forall r\left((r \wedge C r) \rightarrow \exists t_{2} \diamond\left(\operatorname{Present}\left(t_{2}\right) \wedge r \wedge\right.\right.\right.\right.$ $p))))$

Given the Barcan and Converse Barcan Formulas for ' $\square$ ' (i.e. $\square \forall x F x \leftrightarrow \forall x \square F x)^{24}$ and Eternal Temporality $(\square \forall x(\operatorname{Time}(x) \rightarrow \forall t$ at $t(\operatorname{Time}(x))))$, we can move the quantifiers over times in (12) to inside the scope of the modal operators, to get:

(13) $\forall q\left((q \wedge C q) \rightarrow \diamond\left(\exists t_{1}\left(\operatorname{Present}\left(t_{1}\right)\right) \wedge q \wedge \forall r\left((r \wedge C r) \rightarrow \diamond\left(\exists t_{2}\left(\operatorname{Present}\left(t_{2}\right)\right) \wedge r \wedge\right.\right.\right.\right.$ p))))

\footnotetext{
${ }^{24}$ Contingentists - according to whom there could be things that could have been nothing - may find this assumption controversial on the interpretation of ' $\square$ ' as expressing metaphysical necessity. However, see Williamson (2013) for an argument against Contingentism. Note also that ' $\square$ ' does not have to be interpreted as expressing metaphysical necessity here; it could simply be interpreted as expressing necessitation by what propositions there are, for which $\mathrm{BF}$ and $\mathrm{CBF}$ should be automatic whether of not they true for metaphysical necessity.
} 
To show that (13) entails ' $\mathrm{S} p$ ', we must make the following three assumptions:

(i) ' $\square$ ' obeys the modal axiom '4' $(\forall p(\square p \rightarrow \square \square p))$

(ii) ' $\square$ ' obeys the Converse Barcan Formula, or 'CBF' ( $\forall \forall x F x \rightarrow \forall x \square F x$ )

(iii) For any $p$, if $p$ is not about fundamental presentness then necessarily, $p$ is not about fundamental presentness $(\forall p(C p \rightarrow \square C p))$

Given that CBF implies $\diamond \forall x F x \rightarrow \forall x \diamond F x$, we can move the quantifier ' $\forall r$ ' in (13) to outside the scope of the initial ' $\diamond$ ', to get:

$$
\begin{aligned}
& \text { (14) } \mathrm{SS} p \vDash \forall q\left(( q \wedge C ( q ) ) \rightarrow \forall r \diamond \left(\exists t_{1}\left(\operatorname{Present}\left(t_{1}\right)\right) \wedge q \wedge((r \wedge C(r)) \rightarrow\right.\right. \\
& \left.\left.\left.\diamond\left(\exists t_{2}\left(\operatorname{Present}\left(t_{2}\right)\right) \wedge r \wedge p\right)\right)\right)\right)
\end{aligned}
$$

Next, we instantiate $r$ with $q$ to get:

$$
\begin{aligned}
& \text { (15) } \mathrm{SS} p \vDash \forall q\left(( q \wedge C ( q ) ) \rightarrow \diamond \left(\exists t_{1}\left(\operatorname{Present}\left(t_{1}\right)\right) \wedge q \wedge((q \wedge C(q)) \rightarrow\right.\right. \\
& \left.\left.\left.\diamond\left(\exists t_{2}\left(\operatorname{Present}\left(t_{2}\right)\right) \wedge q \wedge p\right)\right)\right)\right)
\end{aligned}
$$

By assumption (iii) above we also have $\forall p(C p \rightarrow \square C p)$, so we can drop the second instance of ' $\mathrm{Cq}$ ' in the above to get:

$$
\begin{aligned}
& \text { (16) } \operatorname{SS} p \vDash \forall q\left(( q \wedge C ( q ) ) \rightarrow \diamond \left(\exists t _ { 1 } ( \operatorname { P r e s e n t } ( t _ { 1 } ) ) \wedge q \wedge \left(q \rightarrow \diamond \left(\exists t_{2}\left(\operatorname{Present}\left(t_{2}\right)\right) \wedge q \wedge\right.\right.\right.\right. \\
& p))))
\end{aligned}
$$


By the basic modal logic ' $\mathrm{K}$ ', (16) implies:

(17) $\operatorname{SS} p \vDash \forall q((q \wedge C(q)) \rightarrow \diamond \diamond(\exists t(\operatorname{Present}(t)) \wedge q \wedge p))$

Finally, by the modal axiom '4', (17) implies:

(18) $\mathrm{SS} p \vDash \forall q((q \wedge C(q)) \rightarrow \diamond(\exists t(\operatorname{Present}(t)) \wedge q \wedge p))$

As ' $\forall q((q \wedge C(q)) \rightarrow \diamond(\exists t(\operatorname{Present}(t)) \wedge q \wedge p))$ ' is equivalent to ' $\mathrm{S} p$ ' given the Modal Analysis, we have shown that the Modal Analysis secures the (necessary) truth of Sometimes Elimination.

\section{Conclusion}

MST is not for everyone. Some philosophers reject Permanentism on the grounds that e.g. Queen Nefertiti is now nothing; some philosophers reject Spotlight on the grounds that every fundamental property is permanent. However, some Permanentists may be sympathetic to the idea that there are temporary fundamental properties. They face a question: if there are such properties, are there very many, or just a few, or exactly one? The answer according to MST is: exactly one. This may seem like an oddly specific answer. Why would anyone who thinks that there is at least one temporary fundamental property be so opinionated as to think that the number of such properties is exactly one? This paper can be seen as providing an answer to that question: because it makes available a certain attractive reductive analysis of the tense 
operators (i.e. the Modal Analysis). In other words, given Spotlight, Permanentists can provide a reductive analysis of change over time. ${ }^{25}$

\section{References}

Bacon, Andrew 2018, 'The Broadest Necessity', Journal of Philosophical Logic 47: 733-83 2016, 'Tense and Relativity', Noûs 52: 667-696

Barcan, Ruth 1946, 'A Functional Calculus of First Order Based on Strict Implication', Journal of Symbolic Logic 11: 1-16

Bigelow, John 1988, The Reality of Numbers: A Physicalist's Philosophy of Mathematics (Oxford: Oxford University Press)

Brogaard, Berit 2012, Transient Truths: An Essay in the Metaphysics of Propositions (Oxford: Oxford University Press)

Cameron, Ross 2015, The Moving Spotlight: An Essay on Time and Ontology (Oxford: Oxford University Press)

Deasy, Daniel 2015, ‘The Moving Spotlight Theory’, Philosophical Studies 172: 2073-89

Dorr, Cian 2019, 'Natural Properties', in Stanford Encyclopedia of Philosophy (Fall 2019

Edition) $<$ https://plato.stanford.edu/archives/fall2019/entries/natural-properties/> 2016, 'To Be F is To Be G', Philosophical Perspectives 30: 39-134 2007, 'What We Disagree About When We Disagree About Ontology', in M. E. Kalderon (ed.), Fictionalism in Metaphysics (Oxford: Oxford University Press), 234-86

\footnotetext{
${ }^{25}$ This paper is dedicated to the memory of Josh Parsons. Acknowledgements: I am grateful to Andrew Bacon for encouraging me to think about the issues addressed in this paper, and to Luca Banfi, Alexander Kaisermann and Thomas Sattig for helpful comments on earlier drafts of the paper. I am also grateful to two anonymous referees for this journal, whose comments helped improve the paper significantly. Finally, I could not have written this paper without the help of Cian Dorr, who provided extensive comments on multiple drafts, responded to vast numbers of emails, made two Zoom calls, and bought me coffee and mince pies in Fellini's Tea Room, Carey's Lane, Cork.
} 
2004, 'Non-symmetric Relations', in D. Zimmerman (ed.), Oxford Studies in

Metaphysics Volume I (Oxford: Oxford University Press), 155-92

Dorr, Cian \& Jeremy Goodman, 2020, 'Diamonds are Forever', Noûs 54: 632-65

Fritz, Peter \& Jeremy Goodman, 2016, 'Higher-order Contingentism Part 1: Closure and

Generation', Journal of Philosophical Logic 45: 645-95

Hossack, Keith, 2007, The Metaphysics of Knowledge (Oxford: Oxford University Press)

Lewis., David K, 1994, 'Humean Supervenience Debugged', Mind 103: 473-90 1986, On the Plurality of Worlds (Oxford: Basil Blackwell) 1983, 'New Work for a Theory of Universals', Australasian Journal of Philosophy 61:

$343-377$

1973, Counterfactuals (Oxford: Basil Blackwell)

Parsons, Josh, 2002, 'A-theory for B-theorists', The Philosophical Quarterly 52: 1-20

Prior, Arthur 1971, Objects of Thought (Oxford: Oxford University Press) 1968, Papers on Time and Tense (Oxford: Oxford University Press)

Prior, Arthur \& Kit Fine, 1977, Worlds, Times, and Selves (London: Duckworth)

Sider, Theodore, 2017, 'Ross Cameron's The Moving Spotlight', Analysis Reviews 77: 78899 2011, Writing the Book of the World (Oxford: Oxford University Press) 2001, Four-dimensionalism: An Ontology of Persistence and Time (Oxford: Oxford University Press)

Stalnaker, Robert 1968, ‘A Theory of Conditionals', American Philosophical Quarterly: 98112

Williamson, Timothy 2013, Modal Logic as Metaphysics (Oxford: Oxford University Press) 